\title{
A JML Tutorial: \\ Modular Specification and Verification of Functional Behavior for Java (Invited Tutorial)
}

\author{
Gary T. Leavens ${ }^{1}$, Joseph R. Kiniry ${ }^{2}$, and Erik Poll ${ }^{3}$ \\ ${ }^{1}$ Dept. of Computer Science, Iowa State University, Ames, IA 50011 USA \\ leavens@cs.iastate.edu \\ ${ }^{2}$ School of Computer Science and Informatics, University College Dublin, \\ Belfield, Dublin 4, Ireland \\ kiniry@acm.org \\ ${ }^{3}$ Computing Science Department, Radboud University Nijmegen, \\ Toernooiveld 1, 6525 ED Nijmegen, The Netherlands \\ erikpoll@cs.ru.nl
}

JML, the Java Modeling Language, is the lingua franca of researchers working on specification and verification techniques and tools for Java. There are over 23 research groups worldwide working on various aspects of the JML project. These groups have built a large suite of tools for automated checking and verification (see http://jmlspecs.org).

This tutorial will present JML features useful for specifying the functional behavior of sequential Java classes and interfaces. Participants will get hands-on experience writing JML specifications for data types, including pre- and postconditions, frames, invariants, history constraints, ghost and model fields, and specfication inheritance. They will also see how to verify object-oriented code using supertype abstraction for modular Hoare-style reasoning. Finally there will be an exchange of ideas on improving existing JML tools, open research problems, and future directions for research related to JML, including ways to connect JML to various theorem provers.

Acknowledgments. Thanks to all involved in JML. Leavens was funded in part by the US National Science Foundation under grant CCF-0429567. Kiniry and Poll were funded in part by the Information Society Technologies programme of the European Commission, Future and Emerging Technologies under the IST2005-015905 MOBIUS project.

\section{References}

1. Leavens, G.T., Baker, A.L., Ruby, C.: Preliminary design of JML: A behavioral interface specification language for Java. ACM SIGSOFT Software Engineering Notes 31(3), 1-38 (2006)

2. Burdy, L., Cheon, Y., Cok, D.R., Ernst, M.D., Kiniry, J.R., Leavens, G.T., Leino, K.R.M., Poll, E.: An overview of JML tools and applications. International Journal on Software Tools for Technology Transfer 7(3), 212-232 (2005) 